\title{
Closed-Form Expression for the Bit-Error Rate of Spectral-Amplitude-Coding Optical CDMA Systems
}

\author{
Hossam M. H. Shalaby, Senior Member, IEEE
}

\begin{abstract}
The spectral-amplitude-coding optical code-division multiple-access (SAC-OCDMA) technique is a vital candidate for future optical networks. Until now, there has been no exact analysis for the performance of systems using these techniques, and most researchers use upper and/or lower bounds to obtain the bit-error rate (BER) of these systems. In this letter, a more accurate analysis of the statistics, in terms of the mean and variance, of the decision current in SAC-OCDMA receivers is developed. These statistics are used to evaluate the BER of SAC-OCDMA techniques based on the Gaussian approximation method. Furthermore, the derived BER is evaluated numerically, for two different types of SAC-OCDMA codes, and is shown to fit properly between the upper and lower bounds of the BER.
\end{abstract}

Index Terms-Bit-error-rate (BER), Hadamard codes, modified quadratic congruence (MQC) codes, optical code-division multiple access (OCDMA), spectral-amplitude coding (SAC).

\section{INTRODUCTION}

$\mathbf{S}$ PECTRAL-amplitude-coding optical code-division multiple-access (SAC-OCDMA) is a promising, cost effective, multiple-access technique that can be used in future passive-optical networks (PONs), free-space optics (FSO), etc. Developing a rigorous mathematical model for the performance of this technique is of special importance for system designers to be able to set suitable parameters of network design. Unfortunately, until now there is no exact analysis for the performance of such techniques and most of the researchers are using the upper bound offered in [1] for bit-error rate (BER) evaluation. Recently, Noshad and Jamshidi [2] have presented both lower and upper bounds on the BER by using a combinatorics approach for the performance analysis of the codes with fixed cross-correlation used in the SAC-OCDMA systems. In this letter, we show that the approach followed by Noshad and Jamshidi can be extended to derive more accurate expressions for both the mean and variance of the decision current in SAC-OCDMA receivers. This helps in obtaining a closed form expression for the BER of SAC-OCDMA techniques. Our derived BER expression is also evaluated numerically for two different types of SAC-OCDMA codes and is shown to fit properly between the upper and lower bounds given by Noshad and Jamshidi.

Manuscript received April 10, 2012; revised May 21, 2012; accepted May 23, 2012. Date of publication May 30, 2012; date of current version June 20, 2012.

The author is with the Department of Electronics and Communications Engineering, Egypt-Japan University of Science and Technology, Alexandria 21934, Egypt, on leave from the Electrical Engineering Department, Alexandria University, Alexandria 21544, Egypt (e-mail: shalaby@ieee.org).

Color versions of one or more of the figures in this letter are available online at http://ieeexplore.iee.org.

Digital Object Identifier 10.1109/LPT.2012.2201934
The remainder of this letter is organized as follows. In Section II, an expression for the BER of SAC-OCDMA technique is derived theoretically. Section III is devoted for numerical evaluation of the BER expression and comparison to upper and lower bounds expressions. Finally, the conclusion is given in Section IV.

\section{TheOretical ANALYsis}

We consider a synchronous SAC-OCDMA network which is composed of $N$ users (or information sources). The $n$th information source, $n \in\{1,2, \ldots, N\}$, generates an iid binary data sequence $\left\{d_{n}\right\}$, where $d_{n} \in\{0,1\}$ and $\operatorname{Pr}\left\{d_{n}=1\right\}=1 / 2$. This sequence modulates the intensity of incoherent light pulses, emitted from an ideal broadband light source. In addition, each user is assigned a SAC-OCDMA code sequence of length $L$, weight $w$, and cross-correlation $\lambda$, selected from a general SAC-OCDMA code set $\mathcal{C}(L, w, \lambda)$. For example, user $n, n \in\{1,2, \ldots, N\}$, is assigned a code sequence $c_{n}=\left\{c_{n}(1), c_{n}(2), \ldots, c_{n}(L)\right\}$, where $c_{n}(i) \in\{0,1\}$ for any $i \in\{1,2, \ldots, L\}$. This code is used to modulate the intensities of the spectral components of each emitted light pulse [1], [3], [4], [5]. We denote the optical source frequency and bandwidth by $f_{c}$ and $\Delta v$, respectively, and the average user power by $P_{a v}$. In our analysis below, we assume that the desired user is the first user. At the receiver side of the desired user, a balanced detection scheme is used [1], [3], [5]. One branch (e.g., upper) of the balanced detector is used to detect the received spectral components that correspond to the user's code $c_{1}$. The other branch (e.g., lower) is used to detect the received spectral components that correspond to the complement of the user's code $\bar{c}_{1}$. Both photodiodes' output currents are then time averaged, scaled, and subtracted to give the decision current. Finally, this decision current is compared to a threshold $\theta$ to decide on the data.

\section{A. Statistics of Upper-Branch Current}

The resultant incident field on upper-branch photodiode PD1 can be written as:

$$
\begin{aligned}
E_{U}(t) & =\sqrt{\frac{P_{0}}{L}} \sum_{n=1}^{N} \sum_{i=1}^{L} d_{n} g(t) c_{n}(i) c_{1}(i) e^{j\left[\omega_{0} t+i \delta \omega t+\phi_{n i}(t)\right]} \\
& =e^{j \omega_{0} t} \sqrt{\frac{P_{0}}{L}} \sum_{n=1}^{N} \sum_{i=1}^{L} d_{n} g(t) c_{n}(i) c_{1}(i) e^{j\left[i \delta \omega t+\phi_{n i}(t)\right]}
\end{aligned}
$$

where $P_{0}=2 P_{a v}, \delta \omega=2 \pi \Delta v / L, f_{0}=f_{c}-\Delta v / 2-\Delta v / L$, $g(t)$ is a normalized envelope of a single-user light field of spectral width $\Delta v / L$, and $\phi_{n i}(t), n \in\{1,2, \ldots, N\}$, 
$i \in\{1,2, \ldots, L\}$, is the phase of the $i$ th chip optical signal of the $n$th user, assumed to be a Wiener process [2] and [6]. We assume that $g(t)$ has the following property:

$$
\frac{1}{T_{b}} \int_{0}^{T_{b}} g^{2}(t) d t=1
$$

where $T_{b}$ is the bit time duration. If the photodiode responsivity is denoted by $\mathcal{R}$, then the average current of PD1 is given by

$$
I_{U}=\frac{\mathcal{R}}{T_{b}} \int_{0}^{T_{b}}\left|E_{U}(t)\right|^{2} d t .
$$

Using (1) and (3), and noticing that the cross-terms cancel out because of the averaging process (as long as $T_{b}>L / \Delta \nu$ ), we get:

$$
\begin{aligned}
I_{U}= & \frac{\mathcal{R} P_{0}}{L} \sum_{i=1}^{L} \frac{1}{T_{b}} \\
& \times \int_{0}^{T_{b}} g^{2}(t)\left|\sum_{n=1}^{N} d_{n} c_{n}(i) c_{1}(i) e^{j \phi_{n i}(t)}\right|^{2} d t .
\end{aligned}
$$

Expanding the last equation, we get

$$
\begin{aligned}
I_{U}= & \frac{\mathcal{R} P_{0}}{L} \sum_{i=1}^{L}\left[\sum_{n=1}^{N} d_{n} c_{n}(i) c_{1}(i)\right. \\
& +2 \sum_{n=1}^{N-1} \sum_{m=n+1}^{N} d_{n} d_{m} c_{n}(i) c_{m}(i) c_{1}(i) \\
& \left.\times \frac{1}{T_{b}} \int_{0}^{T_{b}} g^{2}(t) \cos \left(\phi_{n i}(t)-\phi_{m i}(t)\right) d t\right] .
\end{aligned}
$$

Making use of the properties of $\mathcal{C}(L, w, \lambda)$ code, we get

$$
\begin{aligned}
I_{U}= & \frac{\mathcal{R} P_{0}}{L}\left[w d_{1}+\lambda \sum_{n=2}^{N} d_{n}\right. \\
& +2 \sum_{n=1}^{N-1} \sum_{m=n+1}^{N} d_{n} d_{m} \sum_{i=1}^{L} c_{n}(i) c_{m}(i) c_{1}(i) \\
& \left.\times \frac{1}{T_{b}} \int_{0}^{T_{b}} g^{2}(t) \cos \left(\phi_{n i}(t)-\phi_{m i}(t)\right) d t\right] .
\end{aligned}
$$

We define the following random variable for any $i \in\{1,2, \ldots, L\}$ and any $n, m \in\{1,2, \ldots, N\}$ with $n \neq m$ :

$$
X_{n m}^{i} \stackrel{\text { def }}{=} \frac{1}{T_{b}} \int_{0}^{T_{b}} g^{2}(t) \cos \left(\phi_{n i}(t)-\phi_{m i}(t)\right) d t .
$$

Substituting in (6), we get

$$
\begin{aligned}
I_{U}= & \frac{\mathcal{R} P_{0}}{L}\left[w d_{1}+\lambda \sum_{n=2}^{N} d_{n}\right. \\
& \left.+2 \sum_{n=1}^{N-1} \sum_{m=n+1}^{N} d_{n} d_{m} \sum_{i=1}^{L} c_{n}(i) c_{m}(i) c_{1}(i) X_{n m}^{i}\right] .
\end{aligned}
$$

Using [2] and [6], the average and variance of $X_{n m}^{i}$ are

$$
\begin{aligned}
& \mu_{X_{n m}^{i}} \stackrel{\text { def }}{=} E\left\{X_{n m}^{i}\right\}=0 \\
& \sigma_{X_{n m}^{i}}^{2} \stackrel{\text { def }}{=} \operatorname{var}\left\{X_{n m}^{i}\right\}
\end{aligned}
$$

$$
=\frac{1}{2 T_{b}^{2}} \int_{0}^{T_{b}} \int_{0}^{T_{b}} g^{2}(t) g^{2}(s) e^{-2 \frac{|t-s|}{\tau_{c}}} d t d s
$$

respectively, where $\tau_{c}$ is the coherence time. For the special case where $g(t)$ is a rectangular envelope (9) reduces to

$$
\sigma_{X_{n m}^{i}}^{2} \approx \frac{\tau_{c}}{2 T_{b}}=B_{e} \tau_{c}
$$

where $B_{e}$ is the receiver electrical bandwidth. To get the average and variance of $I_{U}$, we use (9) and (10) in (8), taking into account the independence of the random variables $X_{n m}^{i}$ :

$$
\begin{aligned}
\mu_{I_{U}} \stackrel{\text { def }}{=} & E\left\{I_{U}\right\}=\frac{\mathcal{R} P_{0}}{L}\left(w d_{1}+\lambda \sum_{n=2}^{N} d_{n}\right) \\
= & \frac{\mathcal{R} P_{0}}{L}\left(w d_{1}+\lambda \kappa\right) \\
\sigma_{I_{U}}^{2} \stackrel{\text { def }}{=} & \operatorname{var}\left\{I_{U}\right\}=4 B_{e} \tau_{c}\left(\frac{\mathcal{R} P_{0}}{L}\right)^{2}\left[\sum_{m=2}^{N} d_{1} d_{m} \sum_{i=1}^{L} c_{m}(i) c_{1}(i)\right. \\
& \left.+\sum_{n=2}^{N-1} \sum_{m=n+1}^{N} d_{n} d_{m} \sum_{i=1}^{L} c_{n}(i) c_{m}(i) c_{1}(i)\right] \\
= & 4 B_{e} \tau_{c}\left(\frac{\mathcal{R} P_{0}}{L}\right)^{2}\left[\lambda \sum_{n=2}^{N} d_{1} d_{n}+\frac{\lambda w}{L} \sum_{n=2}^{N-1} \sum_{m=n+1}^{N} d_{n} d_{m}\right] \\
= & 4 B_{e} \tau_{c}\left(\frac{\mathcal{R} P_{0}}{L}\right)^{2}\left[\lambda d_{1} \kappa+\frac{\lambda w}{L} \cdot \frac{\kappa(\kappa-1)}{2}\right] .
\end{aligned}
$$

Here we have made use of the SAC-OCDMA code properties and defined the interference random variable $\kappa$ as:

$$
\kappa \stackrel{\text { def }}{=} \sum_{n=2}^{N} d_{n} .
$$

Of course $\kappa$ follows a binomial distribution:

$$
P_{\kappa}(\ell)=\frac{1}{2^{N-1}}\left(\begin{array}{c}
N-1 \\
\ell
\end{array}\right), \quad \ell \in\{0,1, \ldots, N-1\} .
$$

\section{B. Statistics of Lower-Branch Current}

The resultant incident field on lower-branch photodiode PD2 can be written as:

$$
\begin{aligned}
E_{L}(t)= & \sqrt{\frac{\lambda}{w-\lambda} \cdot \frac{P_{0}}{L}} \sum_{n=1}^{N} \sum_{i=1}^{L} d_{n} g(t) c_{n}(i) \bar{c}_{1}(i) \\
& \times e^{j\left[\omega_{0} t+i \delta \omega t+\phi_{n i}(t)\right]} .
\end{aligned}
$$

It should be noticed that $E_{L}(t)$ is independent of $E_{U}(t)$, as the chips selected by $\left\{\bar{c}_{1}(i)\right\}$ and $\left\{c_{1}(i)\right\}$ are disjoint. Following a similar analysis as we did in the last section, we get the average and variance of lower-branch decision current $I_{L}$ as:

$$
\begin{aligned}
\mu_{I_{L}} \stackrel{\text { def }}{=} & E\left\{I_{L}\right\}=\frac{\mathcal{R} P_{0}}{L} \lambda \sum_{n=2}^{N} d_{n}=\frac{\mathcal{R} P_{0}}{L} \lambda \kappa \\
\sigma_{I_{L}}^{2} \stackrel{\text { def }}{=} & \operatorname{var}\left\{I_{L}\right\}=4 B_{e} \tau_{c}\left(\frac{\lambda}{w-\lambda} \cdot \frac{\mathcal{R} P_{0}}{L}\right)^{2} \\
& \times \sum_{n=2}^{N-1} \sum_{m=n+1}^{N} d_{n} d_{m} \sum_{i=1}^{L} c_{n}(i) c_{m}(i) \bar{c}_{1}(i) \\
= & 4 B_{e} \tau_{c}\left(\frac{\lambda}{w-\lambda} \cdot \frac{\mathcal{R} P_{0}}{L}\right)^{2}\left(\lambda-\frac{\lambda w}{L}\right) \frac{\kappa(\kappa-1)}{2}
\end{aligned}
$$

respectively. 


\section{Statistics of Decision Current}

From the discussion in the above sections, the decision current $I=I_{U}-I_{L}$ has the following mean and variance, given desired user bit $d_{1}=b \in\{0,1\}$ and interference $\kappa=\ell \in\{0,1, \ldots, N-1\}$,

$$
\begin{aligned}
\mu_{b} & =\mu_{I_{U}}-\mu_{I_{L}}=\frac{\mathcal{R} P_{0}}{L} w b \\
\sigma_{b, \ell}^{2} & =\sigma_{P I I N \mid b, \ell}^{2}+\sigma_{s \mid b, \ell}^{2}+\sigma_{T}^{2}
\end{aligned}
$$

respectively, where $\sigma_{b, P I I N}^{2}, \sigma_{s \mid b, \ell}^{2}$, and $\sigma_{T}^{2}$ are the phaseinduced intensity noise (PIIN), shot noise, and thermal noise variances, respectively. These are given by

$$
\begin{aligned}
\sigma_{P I I N \mid b, \ell}^{2}= & \sigma_{I_{U}}^{2}+\sigma_{I_{L}}^{2}=4 B_{e} \tau_{c}\left(\frac{\mathcal{R} P_{0}}{L}\right)^{2}[\lambda b \ell \\
& \left.+\frac{\ell(\ell-1)}{2}\left\{\frac{\lambda w}{L}+\left(\frac{\lambda}{w-\lambda}\right)^{2}\left(\lambda-\frac{\lambda w}{L}\right)\right\}\right] \\
\sigma_{s \mid b, \ell}^{2}= & 2 e B_{e}\left(\mu_{I_{U}}+\mu_{I_{L}}\right)=2 e B_{e} \frac{\mathcal{R} P_{0}}{L}(w b+2 \lambda \ell) \\
\sigma_{T}^{2}= & 4 k_{B} T^{\circ} B_{e} / R_{L}
\end{aligned}
$$

respectively. Here $e=1.6 \times 10^{-19} \mathrm{C}$ is the electron charge, $k_{B}=1.38 \times 10^{-23} \mathrm{~J} / \mathrm{K}$ is Boltzmann's constant, $T^{\circ}$ is the receiver noise temperature, and $R_{L}$ is the receiver load resistor.

\section{BER Evaluation}

The bit error probability $P_{e}$ can be written as

$$
P_{e}=\frac{1}{2} \sum_{\ell=0}^{N-1}\left[P_{e \mid \kappa, d_{1}}(\ell, 1)+P_{e \mid \kappa, d_{1}}(\ell, 0)\right] P_{\kappa}(\ell)
$$

where $P_{e \mid \kappa, d_{1}}(\ell, b)$ is the conditional BER given $\kappa=\ell \in$ $\{0,1, \ldots, N-1\}$ interference and that the desired user has sent data bit $d_{1}=b \in\{0,1\}$, which is evaluated as

$$
\begin{aligned}
P_{e \mid \kappa, d_{1}}(\ell, 1) & =\frac{1}{\sigma_{1, \ell} \sqrt{2 \pi}} \int_{-\infty}^{\theta} e^{\frac{\left(y-\mu_{1}\right)^{2}}{2 \sigma_{1, \ell}^{2}}} d y \\
& =\frac{1}{2} \operatorname{erfc}\left(\frac{\mu_{1}-\theta}{\sigma_{1, \ell} \sqrt{2}}\right) \\
P_{e \mid \kappa, d_{1}}(\ell, 0) & =\frac{1}{\sigma_{0, \ell} \sqrt{2 \pi}} \int_{\theta}^{\infty} e^{\frac{\left(y-\mu_{0}\right)^{2}}{2 \sigma_{0, \ell}^{2}}} d y \\
& =\frac{1}{2} \operatorname{erfc}\left(\frac{\theta-\mu_{0}}{\sigma_{0, \ell} \sqrt{2}}\right)
\end{aligned}
$$

where $\theta$ is the threshold. Substituting in (18), we get

$$
P_{e}=\frac{1}{4} \sum_{\ell=0}^{N-1} \frac{\left(\begin{array}{c}
N-1 \\
\ell
\end{array}\right)}{2^{N-1}}\left[\operatorname{erfc}\left(\frac{\mu_{1}-\theta}{\sigma_{1, \ell} \sqrt{2}}\right)+\operatorname{erfc}\left(\frac{\theta-\mu_{0}}{\sigma_{0, \ell} \sqrt{2}}\right)\right] .
$$

\section{NUMERICAL RESULTS}

The bit-error rate (BER) as given in (20) has been evaluated numerically for both modified quadratic congruence (MQC) and Hadamard codes. The length, weight, and cross-correlation $(L, w, \lambda)$ of these codes are $(132,12,1)$ and $(128,64,32)$, respectively. Both BERs are plotted in Fig. 1 versus different

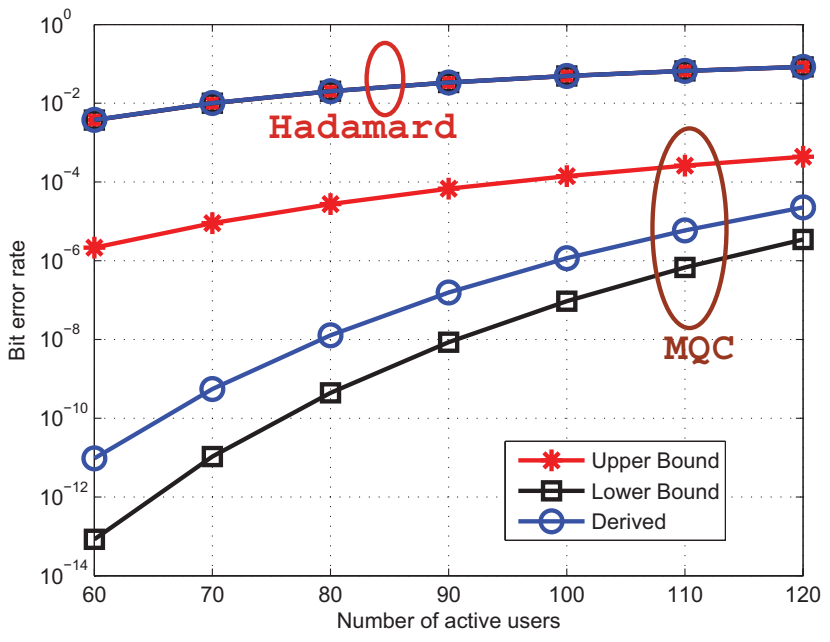

Fig. 1. SAC-CDMA BER comparison with Noshad and Jamshidi bounds, using MQC $(132,12,1)$ and Hadamard $(128,64,32)$ codes.

numbers of active users. Other parameters used in the evaluations are as follows. The data bit rate $R_{b}=130 \mathrm{Mb} / \mathrm{s}$, the linewidth of the light source $\Delta v=3.5 \mathrm{THz}$, the coherence time $\tau_{c}=L / \Delta v=37.714 \mathrm{ps}$, the receiver noise temperature $T^{\circ}=300 \mathrm{~K}$, the receiver load resistance $R_{L}=1 \mathrm{k} \Omega$, the photodiode responsivity $\mathcal{R}=1$, and the average received power $P_{0}=-10 \mathrm{dBm}$. Upper and lower bounds of the BER, as derived in [2] are depicted on the same figure as well. It is clear from the figure that the BER as given in (20) fits well between both bounds and behaves similarly to the simulation results as given in [2]. Notice that in the case of Hadamard codes, both BER bounds as well as our derived BER coincide with each other. Indeed, it has been shown in [2] that both the upper and lower bounds are equal in this case.

\section{CONCLUSION}

A more accurate analysis of the statistics, in terms of the mean and variance, of the decision current in SAC-OCDMA receivers has been developed. Based on these statistics, a Gaussian approximation of the BER has been obtained. The derived BER has been evaluated numerically and compared to upper and lower bounds of the BER for two different SAC-OCDMA codes.

\section{REFERENCES}

[1] E. D. J. Smith, R. J. Blaikie, and D. P. Taylor, "Performance enhancement of spectral-amplitude-coding optical CDMA using pulse-position modulation," IEEE Trans. Commun., vol. 46, no. 9, pp. 1176-1185, Sep. 1998.

[2] M. Noshad and K. Jamshidi, "Bounds for the BER of codes with fixed cross correlation in SAC-OCDMA systems," J. Lightw. Technol., vol. 29, no. 13, pp. 1944-1950, Jul. 1, 2011.

[3] D. Zaccarin and M. Kavehrad, "An optical CDMA system based on spectral encoding of LED," IEEE Photon. Technol. Lett., vol. 4, no. 4, pp. 479-482, Apr. 1993.

[4] X. Zhou, H. M. H. Shalaby, and C. Lu, "Design and performance analysis of a new code for spectral-amplitude-coding optical CDMA systems," in Proc. IEEE 6th ISSSTA, Sep. 2000, pp. 174-178.

[5] Z. Wei, H. M. H. Shalaby, and H. Ghafouri-Shiraz, "Modified quadratic congruence codes for fiber Bragg-grating-based spectral-amplitudecoding optical CDMA systems," J. Lightw. Technol., vol. 19, no. 9, pp. 1274-1281, Sep. 2001.

[6] M. M. Rad and J. A. Salehi, "Phase-induced intensity noise in digital incoherent all-optical tapped-delay line systems," J. Lightw. Technol., vol. 24, no. 8, pp. 3059-3072, Aug. 2006. 